\title{
ON THE ZEROS OF SUCCESSIVE DERIVATIVES
}

\section{ALBERT EDREI}

1. Statement of the main result. The following analogue of a theorem of Landau [1, p. 102] seems to be new.

Theorem 1a. Consider the class $\mathcal{F}_{\alpha}$ of all analytic functions $f$, regular at the origin and such that

$$
1=f(0)=f^{\prime}(0) ; \quad \alpha=f^{\prime \prime}(0) \neq 1 .
$$

There exists a positive function $R_{1}(\alpha)$, finite for

$$
\alpha \neq 1 \text {, }
$$

and such that every function $f\left(\in \mathcal{F}_{\boldsymbol{\alpha}}\right)$ has, in the region

$$
|z| \leqq R_{1}(\alpha) \text {, }
$$

either a singularity or else a zero of

$$
f(z) f^{\prime}(z) f^{\prime \prime}(z) \text {. }
$$

The function $R_{1}(\alpha)$ may be chosen as follows:

$$
R_{1}(\alpha)=\max \left\{A_{1}, \frac{A_{2}}{|\alpha-1|} \log \frac{1}{|\alpha-1|}\right\} \quad \text { for }|\alpha| \leqq A_{3},
$$

(1.6a) $\quad R_{1}(\alpha)=A_{4} \frac{\log |\alpha|}{|\alpha|}$

where $A_{1}, A_{2}, A_{3}, A_{4}$ denote suitable numerical constants (independent of $f$ ).

Instead of proving Theorem 1a, we shall prove the following closely related result.

Theorem $1 \mathrm{~b}$. Consider the class $G_{\beta}$ of all analytic functions $g$, regular at the origin and such that

$$
1=g(0) ; \quad \beta=g^{\prime}(0) \neq 0 .
$$

There exists a positive function $R_{2}(\beta)$, finite for

$$
\beta \neq 0 \text {, }
$$

and such that every function $g\left(\in G_{\beta}\right)$ has, in the region

Presented to the Society, December 28, 1953; received by the editors May 7, 1954 and, in revised form, July 20, 1954. 


$$
|z| \leqq R_{2}(\beta),
$$

either a singularity or else a zero of

$$
g(z)\left[g^{\prime}(z)-1\right] \text {. }
$$

The function $R_{2}(\beta)$ may be chosen as follows:

$$
\begin{array}{ll}
R_{2}(\beta)=\max \left[B_{1}, \frac{B_{2}}{|\beta|} \log \frac{1}{|\beta|}\right] & \text { for }|\beta| \leqq B_{3}, \\
R_{2}(\beta)=B_{4} \frac{\log |\beta|}{|\beta|} & \text { for }|\beta|>B_{3},
\end{array}
$$

where $B_{1}, B_{2}, B_{3}, B_{4}$ denote suitable numerical constants (independent of $g$ ).

Theorem $1 \mathrm{a}$ is immediately obtained by applying Theorem $1 \mathrm{~b}$ to the function

$$
g(z)=\frac{f(z)}{f^{\prime}(z)} .
$$

2. Remarks on the structure of Theorem 1a. Our definitions imply that if $f \in \mathcal{F}_{\alpha}$, the expansion

$$
f(z)=1+z+\frac{\alpha}{2 !} z^{2}+\cdots,
$$

has a positive (or infinite) radius of convergence $\rho[f]$.

Now let $R[f]$ be a non-negative number defined as follows:

(i) $R[f]=\rho[f]$ if $f(z) f^{\prime}(z) f^{\prime \prime}(z) \neq 0$ for $|z|<\rho[f]$;

(ii) otherwise, let $z_{0}$ denote the zero (or one of the zeros) of least modulus of the function $f(z) f^{\prime}(z) f^{\prime \prime}(z)$ and put

$$
R[f]=\left|z_{0}\right| \text {. }
$$

For certain special choices of $f$ it is possible to determine $R[f]$ explicitly. For instance, if $\alpha \neq 1$,

$$
R\left[\exp \left\{\frac{e^{(\alpha-1) z}-1}{\alpha-1}\right\}\right]=\left|\frac{\log (1-\alpha)}{1-\alpha}\right|,
$$

where the determination of the logarithm is chosen so as to minimize $|\log (1-\alpha)|$.

If $\alpha \rightarrow 1,(2.1)$ takes the limiting form

$$
R\left[e^{z}\right]=+\infty,
$$


which is obviously correct. In connection with (2.2), we observe that if $h(z)$ is entire and if $h h^{\prime} h^{\prime \prime}$ never vanishes, Theorem 1a shows that $h$ must satisfy the differential equation $h h^{\prime \prime}=\left\{h^{\prime}\right\}^{2}$. Hence, as was proved by Pólya $[3$, p. 38, footnote 1a] and Saxer $[5$, p. 206], $h(z)$ is of the form $\exp (a z+b)$.

Now let $\alpha(\neq 1)$, be fixed. In view of Theorem 1a, the relation

$$
R_{0}(\alpha)=\sup _{\mathcal{f} \in \mathscr{F}_{\alpha}} R[f]
$$

defines a finite quantity $R_{0}(\alpha)$; moreover, in view of (2.3), (2.1), and (1.3a)

$$
\left|\frac{\log (1-\alpha)}{1-\alpha}\right| \leqq R_{0}(\alpha) \leqq R_{1}(\alpha)
$$

It would be of interest to determine exactly $R_{0}(\alpha)$. Although this is perhaps difficult, a suitable modification of our arguments readily yields

$$
\limsup _{\alpha \rightarrow 1} \frac{R_{0}(\alpha)|1-\alpha|}{|\log | 1-\alpha||} \leqq 2 ; \quad \limsup _{\alpha \rightarrow \infty} \frac{R_{0}(\alpha)|\alpha|}{\log |\alpha|} \leqq 2 .
$$

3. Applications of Theorem 1a. The limit points of the zeros of the derivatives of an analytic function are usually difficult to investigate. The two theorems which we state and prove in this section will show how Theorem 1a may be used in such investigations.

Let $z_{0}$ be a point of regularity of $h(z)$ and assume

$$
h^{(n)}\left(z_{0}\right) h^{(n+1)}\left(z_{0}\right) \neq 0, \quad \alpha=\frac{h^{(n)}\left(z_{0}\right) h^{(n+2)}\left(z_{0}\right)}{\left\{h^{(n+1)}\left(z_{0}\right)\right\}^{2}} \neq 1 .
$$

Put

$$
t=\left(z-z_{0}\right) \frac{h^{(n+1)}\left(z_{0}\right)}{h^{(n)}\left(z_{0}\right)}
$$

and consider the function

$$
\phi(t)=\frac{h^{(n)}\left(z_{0}+t\left(h^{(n)}\left(z_{0}\right) / h^{(n+1)}\left(z_{0}\right)\right)\right)}{h^{(n)}\left(z_{0}\right)}=1+t+\alpha \frac{t^{2}}{2 !}+\cdots
$$

Applying Theorem 1a to this function, we obtain bounds for the radius of the largest circle, with center at $z_{0}$, free of singularities of $h(z)$ and of zeros of

$$
h^{(n)}(z) h^{(n+1)}(z) h^{(n+2)}(z)
$$


These considerations obviously yield:

THEOREM 2. Let $h(z)$ be regular at $z_{0}$. Consider a sequence $\left\{n_{\nu}\right\}_{\nu=1}^{\infty}$ of positive integers and the two sequences

$$
\begin{gathered}
\left\{\frac{h^{\left(n_{\nu}+1\right)}\left(z_{0}\right)}{h^{\left(n_{\nu}\right)}\left(z_{0}\right)}\right\}_{\nu=1}^{\infty}, \\
\left\{\frac{h^{\left(n_{\nu}+2\right)}\left(z_{0}\right) h^{\left(n_{\nu}\right)}\left(z_{0}\right)}{\left[h^{\left(n_{\nu}+1\right)}\left(z_{0}\right)\right]^{2}}\right\}_{\nu=1}^{\infty} .
\end{gathered}
$$

The point $z_{0}$ is a limit point of the zeros of the derivatives of $h(z)$ if one of the two following conditions is satisfied:

I. (3.2) tends to infinity and (3.3), if it converges, does not tend to one.

II. (3.3) tends to infinity and (3.2), if it converges, does not tend to zero.

Theorem 2 has the following consequence, which generalizes substantially a result of Rådström [4, p. 138].

TheOREM 3. Let

$$
h^{*}(z)=\sum_{\nu=0}^{\infty} a_{\nu} z^{\nu}
$$

be a function which, if it is entire, is not of exponential type.

It is possible to find a sequence $\left\{\omega_{\nu}\right\}_{\nu=0}^{\infty}$ of numbers, each of which is +1 or -1 and such that the origin is a limit point of the zeros of the derivatives of

$$
h(z)=\sum_{\nu=0}^{\infty} a_{\nu} \omega_{\nu} z^{\nu}
$$

The proof is immediate: we apply Theorem 2 with $z_{0}=0$. As $h^{*}(z)$ is not of exponential type there exists a sequence $\left\{n_{\nu}\right\}$ such that (3.2) tends to infinity. Moreover, it is clear that a suitable choice of $\left\{\omega_{\nu}\right\}$ will prevent (3.3) from converging to 1 .

4. Proof of Theorem 1b. A theorem of Miranda [2, p. 185] contains $^{1}$ the following proposition which we state as

LEMMA 1. Let $h(z)$ be regular for $|z|<1$ and such that

$$
h(z) \neq 0, \quad h^{\prime}(z) \neq 1 .
$$

Then, there exist two positive absolute constants $P$ and $Q$ such that

${ }^{1}$ Cf. Valiron [6, p. 21]. 


$$
m(1 / 2, h)<P \log ^{+}|h(0)|+Q .
$$

Using Nevanlinna's formula [6, p. 12] and Jensen's theorem, (4.1) implies

$$
\begin{aligned}
\left|\frac{h^{\prime}(0)}{h(0)}\right| & \leqq 4[m(1 / 2, h)+m(1 / 2,1 / h)] \\
& <4\left[2 P \log ^{+}|h(0)|+2 Q-\log |h(0)|\right]
\end{aligned}
$$

Let $g(z)$ be a function of the class $G_{\beta}$ and assume that for $|z|<R$, $g(z)$ is regular and

$$
g(z) \neq 0, \quad g^{\prime}(z) \neq 1
$$

Taking

$$
h(z)=\frac{g(R z)}{R}
$$

(4.2) yields

$$
R|\beta|<8 P \log ^{+} \frac{1}{R}+4 \log R+8 Q
$$

We may, if necessary, increase the value of $Q$ so that it is no restriction to assume $Q \geqq 2$. Then, if

$$
\begin{gathered}
|\beta|>8 Q^{2}(\geqq 32), \\
R \geqq 1,
\end{gathered}
$$

(4.3), (4.4), and (4.5) imply

$$
8 Q^{2} R<4 R^{1 / 2}+8 Q .
$$

As (4.6) contradicts (4.5), it is clear that (4.4) implies $R<1$ and hence (4.3) yields

$$
R|\beta|+8 P \log R<8 Q .
$$

Consider the quantity $S$ uniquely defined by

$$
S|\beta|+8 P \log S=8 Q \text {. }
$$

Clearly

$$
\begin{gathered}
R<S<1, \quad S|\beta|>8 Q \\
R<S<\frac{8 Q}{|\beta|}+\frac{8 P}{|\beta|} \log \frac{|\beta|}{8 Q}<\frac{8(P+Q)}{|\beta|} \log |\beta|,
\end{gathered}
$$


so that we may take

$$
B_{3}=8 Q^{2}, \quad B_{4}=8(P+Q) .
$$

We now find an upper bound for $R$ which does not depend on the assumption

$$
|\beta|>B_{3}
$$

We either have $R<e$ or else (4.3) implies

$$
\begin{gathered}
R|\beta|<(4+8 Q) \log R<(4+8 Q) R^{1 / 2}, \\
R<\frac{4+8 Q}{|\beta|} \log \left(\frac{4+8 Q}{|\beta|}\right)^{2} .
\end{gathered}
$$

The determination of suitable constants $B_{1}$ and $B_{2}$ is now trivial.

\section{REFERENCES}

1. E. Landau, Darstellung und Begrilndung einiger neuerer Ergebnisse der Funktionentheorie, 2d ed., Berlin, 1929.

2. C. Miranda, Sur un nouveau critère de normalite pour les familles de fonctions holomorphes, Bull. Soc. Math. France vol. 63 (1935) pp. 185-196.

3. G. Polya, Über die Nullstellen sukzessiver Derivierten, Math. Zeit. vol. 12 (1922) pp. 36-60.

4. H. Rådström, Zeros of successive derivatives, Arkiv för Matematik (1949) pp. 101-139.

5. W. Saxer, Über die Picardschen Ausnahmewerte sukzessiver Derivierten, Math. Zeit. vol. 17 (1923) pp. 206-227.

6. G. Valiron, Sur les valeurs exceptionnelles des fonctions meromorphes et de leurs derivées, Paris, 1937.

Syracuse UNIVERSITY 